\title{
Comparative Study on Facial Expression Recognition using Gabor and Dual-Tree Complex Wavelet Transforms
}

\author{
Alaa Eleyan \\ Electrical \& Electronics Engineering Department, Avrasya University, Yomra, Trabzon, TURKEY \\ E-mail address: aeleyan@avrasya.edu.tr \\ Received date: January 2017
}

\begin{abstract}
Moving from manually interaction with machines to automated systems, stressed on the importance of facial expression recognition for human computer interaction $(\mathrm{HCI})$. In this article, an investigation and comparative study about the use of complex wavelet transforms for Facial Expression Recognition (FER) problem was conducted. Two complex wavelets were used as feature extractors; Gabor wavelets transform (GWT) and dual-tree complex wavelets transform (DT-CWT). Extracted feature vectors were fed to principal component analysis (PCA) or local binary patterns (LBP). Extensive experiments were carried out using three different databases, namely; JAFFE, CK and MUFE databases. For evaluation of the performance of the system, k-nearest neighbor ( $k N N)$, neural networks (NN) and support vector machines (SVM) classifiers were implemented. The obtained results show that the complex wavelet transform together with sophisticated classifiers can serve as a powerful tool for facial expression recognition problem.
\end{abstract}

Keywords: facial expression, complex wavelet transform, local binary pattern, principle component analysis, neural networks, support vector machines.

\section{Introduction}

In recent decades, significant attention of researchers was drawn to the areas of digital image processing, computer vision and pattern recognition. This can be justified as a result of the increase demand of robots in security, entertainment, industry and healthcare related applications $[1,2]$. In the design of HCI [3], robots and humans can communicate in more natural way if the robots are able to understand human gestures and facial expressions [4]. This can make them respond efficiently and harmonically during tasks where both must work together.

Facial expression is simply a contraction of facial muscles that reflects the emotional state of an individual. Facial Expression Recognition (FER) is an application for automatically identifying a face of a person from an image or a video sequence and compares it with the database to interpret the emotional state of that person. Facial expressions play a significant role in nonverbal human communications. According to the Mehrabian formula; 7\%, 38\% and 55\% of message pertaining to feelings and attitudes is in the spoken words, paralinguistic and in facial expression, respectively [5].

Ekman [6], specified a set of six basic emotions; anger, disgust, fear, happy, sad and surprise which are universal and correlated with muscular patterns in all cultures. Later on, the neutral expression was included as the seventh basic emotion. Generally, the existing approaches to facial expression 
recognition is categorized into geometric based approaches [7,8] and appearance based approaches $[9,10]$.

Several algorithms have been introduced and/or improved to reduce the gap between human and computers in accuracy of FER. Currently, Wavelet Transform algorithms such as Discrete Wavelet Transform (DWT), Gabor Wavelet Transform (GWT) and Dual-Tree Complex Wavelet Transform (DT-CWT) are commonly used for feature extraction due to their multi-scale and multi-directional properties. GWT and DT-CWT can demonstrate suitable characteristic of directional selectivity and spatial locality and utilize all the information in space and frequency domain. They also have the advantage of eliminating the effect of non-uniform illumination in a face image.

The DWT is a well-known algorithm for feature extraction. However, it lacks good directional selectivity, shift variance sensitivity and phase information. GWT, on the other hand, can overcome those limitations. It has been extensively used in the field of image processing and computer vision applications. The Gabor wavelet filter is an essential tool used to capture and extract local features both in spatial and frequency domain aligned at particular directions and scales $[11,12]$.

Lyons et al. [13] proposed an approach for automatic facial images classification based on labelled elastic graph matching and GWT. The work conducted in $[14,11]$, claimed that biological relevance of GWT makes it a powerful tool as a feature extractor. Researchers in [15] used GWT as a feature extraction technique for palmprint recognition while in [16] they enhanced the fingerprint images using GWT.

However, GWT suffers from its high computational complexity and huge memory requirement to store the large feature vectors extracted. For instance, for an image of size $256 \times 256$, with a Gabor filter of 5 scales and 8 directions, the 40 magnitude responses reside in $256 \times 256 \times 40(2621440)$ dimensional space which is very huge and requires a large storage capacity.

The popularly known DT-CWT introduced by Kingsbury [17], imitates Gabor wavelet kernels and provides good directional selectivity in 6 fixed directions at different scales. Furthermore, the DTCWT has some added advantages such as its limited redundancy for image and it is much computationally faster than Gabor wavelets; hence it serves as a perfect replacement for Gabor wavelet filter. Thus, with DT-CWT we can get a comparable performance with less computational complexity. Researchers in $[18,19]$ applied DT-CWT to enhance the face recognition performance. Sun et al. [20] used DT-CWT for face detection using spectral histogram. Y. Wang in [21], investigated the application of the DT-CWT based on local binary pattern weighted histogram method for palmprint recognition. In [22], DT-CWT and SVM was used for the image de-noising problem. Moreover, DT-CWT was also used in [23] with SVM for handwritten numeral recognition. Face recognition problem was addressed in [24] using three different complex wavelet transforms. Researchers in [25] used DT-CWT for facial expression recognition together with supervised spectral analysis.

The rest of the article is organized as follows. Sections 2 and 3 gives a brief overview of GWT \& DT-CWT. The two dimensionality reduction algorithms are described in section 4 . Section 5 discusses the proposed approach, while Section 6 gives the simulation results and discussions. Finally, section 7 contains drawn conclusions. 


\section{Complex Wavelets Transforms}

\subsection{Gabor Wavelet Transform}

It is well established that the scales and directions of the GWT are similar to that of the primary visual cortex of the mammalian brain [26]. GWT is widely used in many pattern recognition analyses. The GWT extracts the features of the input image that are aligned at particular directions and scales.

GWT possesses many properties which makes it suitable for different applications. It has good directional selectivity and is insensitive to illumination variations. A 2D Gabor filter can be defined in both spatial and frequency domain as [27]:

$$
\Psi_{u, v}(x, y)=\frac{f_{u}{ }^{2}}{\pi \kappa \eta} e^{-\left(\left(f_{u}{ }^{2} / \kappa^{2}\right) x^{\prime 2}+\left(f_{u}{ }^{2} / \eta^{2}\right) y^{\prime 2}\right)} e^{j 2 \pi f_{u} x^{\prime}}
$$

where $(x, y)$ are the coordinates of the pixel's values, $x^{\prime}=x \cos \theta_{v}+y \sin \theta_{v}, y^{\prime}=-x \sin \theta_{v}+$ $y \cos \theta_{v}$ and $\left(x^{\prime}, y^{\prime}\right)$ are the new coordinates of the pixel values after anti clockwise rotation from the old coordinates $(x, y)$.

$$
\begin{gathered}
f_{u}=f_{\max } / \sqrt[u]{2} \\
\theta_{v}=v \pi / 8
\end{gathered}
$$

$f_{u}$ is the center frequency of the complex sinusoid, $\sqrt[u]{2}$ is the spacing factor between different central frequencies and $\theta_{v}$ is the orientation of the wavelet. The $\kappa$ and $\eta$ are the sharpness along the minor and the major axis of the Gaussian envelop. The commonly used values for face and facial expression recognition are $\kappa=\eta=\sqrt{2}$ and $f_{\max }=0.25$ with a scale of $5(u=0,1,2,3,4)$ and orientation of $8(v=0,1,2,3,4,5,6,7)$ [28,29]. If $\mathbf{I}(x, y)$ is the face image of size $N \times M$ and $\boldsymbol{\Psi}_{u, v}(x, y)$ is the Gabor filter, then the feature extraction which is the filtering operation of the image and the Gabor filter can be defined as:

$$
\mathbf{G}_{u, v}(x, y)=\mathbf{I}(x, y) * \boldsymbol{\Psi}_{u, v}(x, y)
$$

where $\mathbf{G}_{u, v}(x, y)$ represents the output of the filtering process and $*$ is the convolution operator. Fig. 1 shows example of an image to be filtered, magnitude and real parts of the Gabor wavelets filters at 5 scales and 8 directions and the magnitude of the output obtained after filtering.

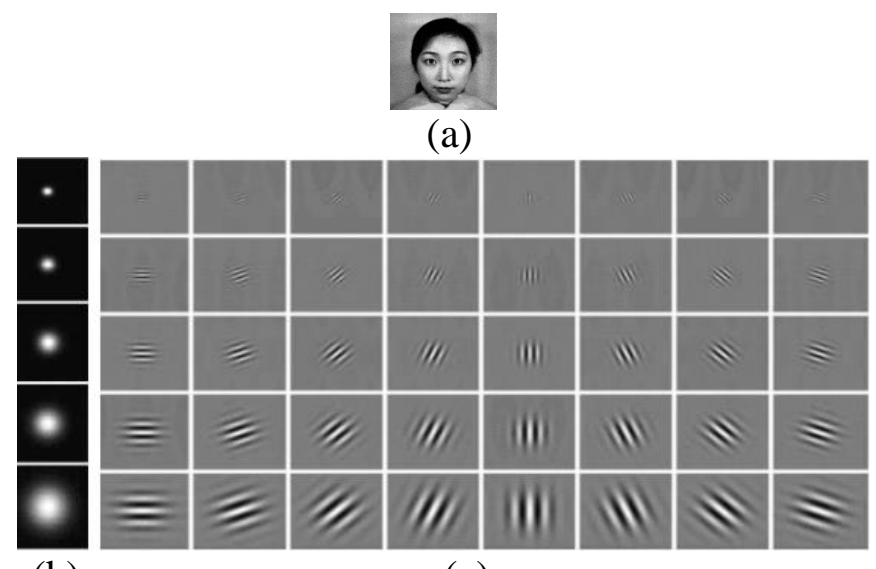

(b)

(c) 


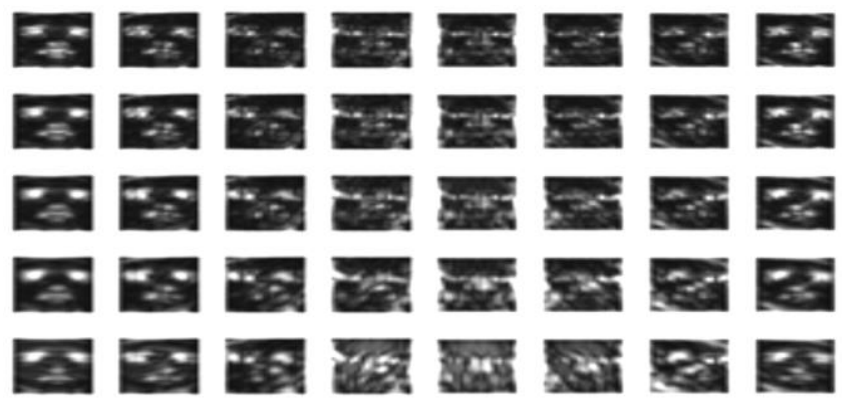

(d)

Fig. 1. (a) Image to be filtered (b) Magnitude of Gabor filter at 5 scales and (c) Real parts at 5 scales and 8 directions of the Gabor wavelets filters and (d) Magnitude of the output obtain after convolution.

\subsection{Dual-Tree Complex Wavelet Transform}

The DT-CWT possesses similar shapes to Gabor Wavelets Transform [17]. As shown in Fig. 2, the DT-CWT contains two trees of real filters, tree ' $a$ ' and tree ' $b$ ', which give the real and imaginary parts of the complex coefficients.

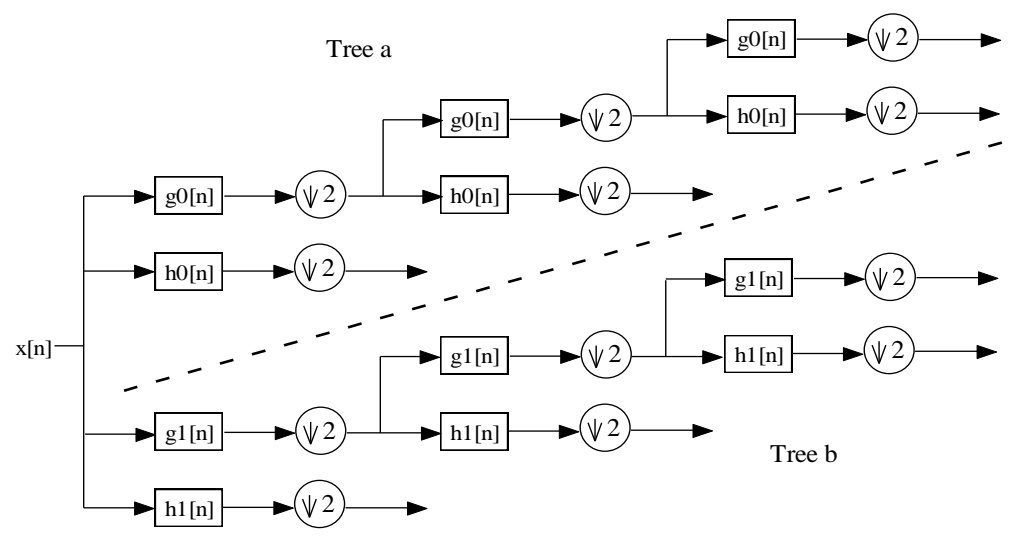

Fig. 2. Dual-Tree Complex Wavelet Transform [17].

Kingsbury summarized the properties of DT-CWT as: approximate shift invariance, good directional selectivity in 2-D with Gabor-like filters, perfect reconstruction using short linear-phase filters, limited redundancy, independent of the number of scales, and efficient order- $\mathrm{N}$ computation.

The DT-CWT filters used are designed to give perfect reconstruction at every scale. The transform has the ability to differentiate positive and negative frequencies and produce six sub-bands strongly oriented in $\pm 15^{\circ}, \pm 45^{\circ}$, and $\pm 75^{\circ}$ as shown in Fig. 3(c). However, unlike Gabor wavelet transform where the sub-band can be computed in any orientation, here the directions are fixed. The DT-CWT expansion of an image $\mathbf{f}(\vec{x})$ is given by:

$$
\mathbf{f}(\vec{x})=\sum_{k} \mathbf{W}_{\varphi}\left(j_{o}, k\right) \boldsymbol{\varphi}_{j_{o}, k}(\vec{x})+\sum_{i} \sum_{j>j_{o}} \sum_{k} \mathbf{W}_{\psi}(j, k) \boldsymbol{\Psi}_{j, k}^{i}(\vec{x}),
$$


where $i= \pm 15^{\circ}, \pm 45^{\circ}$, and $\pm 75^{\circ}$. The scaling function $\boldsymbol{\varphi}_{j_{o}, k}$ and the wavelet function $\boldsymbol{\Psi}_{j, k}^{i}$ are complex. $\mathbf{W}_{\varphi}\left(j_{o}, k\right)$ indicates the scaling coefficients and $\mathbf{W}_{\psi}(j, k)$ are wavelet coefficients of the transform. Fig. 3 shows face image from JAFFE database, the real part and magnitude of impulse response of DT-CWT and the magnitude response obtained for JAFFE face image using DT-CWT at 3 scales and 6 fixed directions $\left( \pm 15^{\circ}, \pm 45^{\circ}\right.$ and $\left.\pm 75^{\circ}\right)$.

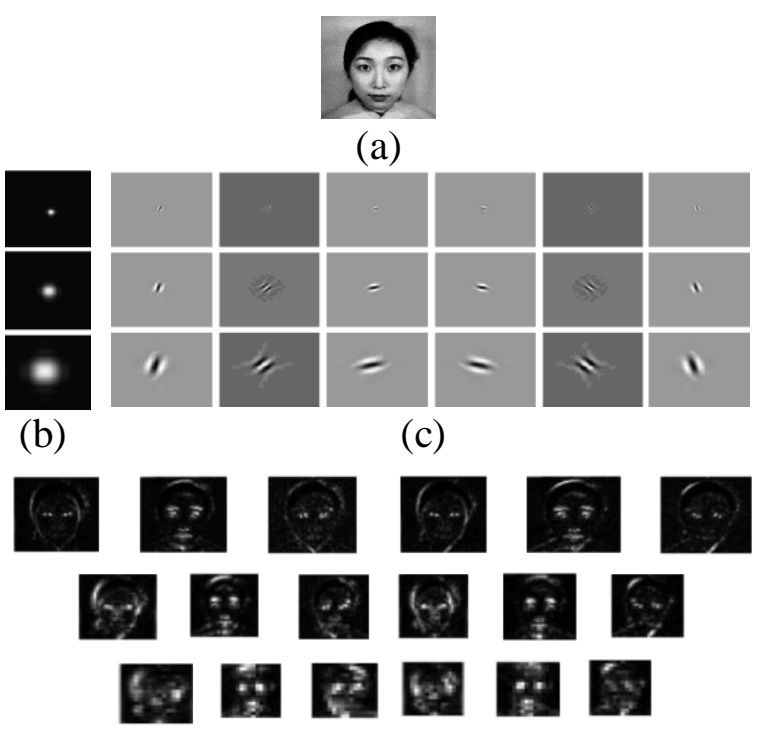

(d)

Fig. 3. (a) Face image from JAFFE database (b) Magnitude of the DT-CWT filter response (c) Real part of DT-CWT filter response at 3 scales and 6 directions. (d) The magnitude response obtained for JAFFE face image using DT-CWT for 3 scales and 6 directions

The dimensions of the feature vectors created by GWT and DT-CWT are huge as such dimensionality reduction is usually employed by linearly combining the feature vectors and projecting the high dimensional features onto a lower dimensional space [28,29].

\section{Dimensionality Reduction}

\subsection{Principal Component Analysis}

Principle Component Analysis (PCA) is one of the classical techniques used to find the effective linear transformations. PCA is extensively used in signal processing and pattern recognition applications for feature extractions and data dimensionality reduction [10]. In this article, PCA was used to reduce the dimensionality of the GWT and DT-CWT feature vectors. For further details regarding PCA algorithm reader should return to [10].

\subsection{Local Binary Patterns}

Even though LBP is a feature extraction algorithm rather than being a tool for dimensionality reduction, we benefited from its working mechanism to reduce the dimensionality of the feature vectors generated using wavelet transforms. The LBP algorithm [30] is dividing the face image into 
non-overlapping regions. For each region, a $3 \times 3$ neighborhood is defined. The neighboring pixels are examined based on the central pixel grayscale value which threshold the neighbors to 1 or 0 . Hence an 8-bit binary string representing each pixel will be formed which will be converted to a decimal number. Histograms of 256 bins or less for every region will be formed based on the obtained decimal numbers. These histograms are then concatenated to form the feature vector for the face image. An example of the basic LBP operator is shown in Fig. 4.

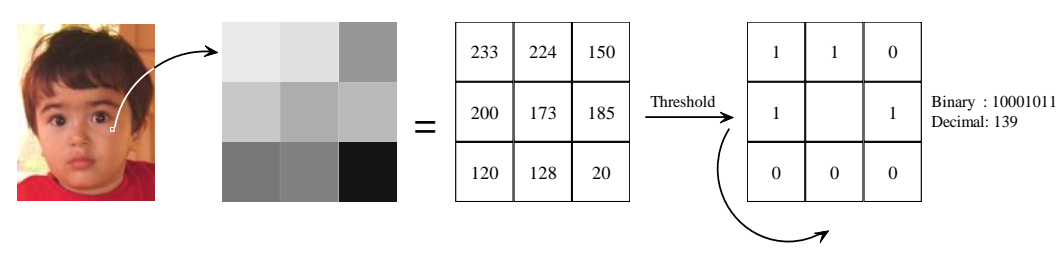

Fig. 4. Example of $3 \times 3$ Basic LBP operators.

\section{The Proposed Approach}

The proposed approach for facial expression recognition is shown in Fig. 5.

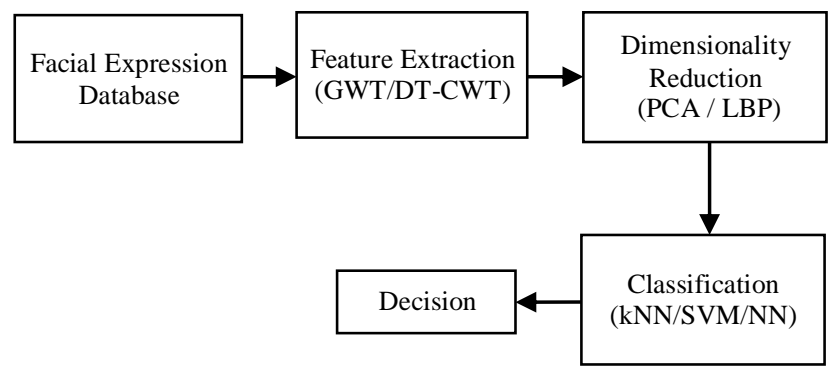

Fig. 5. Block diagram of the proposed approach.

Facial expression database contains the images that will be used both for training and testing. Feature extraction computes salient features (feature vectors) from the face so that redundant information can be discarded. The features of the GWT were extracted using 5 scales and 8 directions. While the DTCWT salient features were extracted using 3 scales and 6 directions. LBP and PCA were used to reduce the dimensionality of feature vectors of GWT and DT-CWT. A uniform LBP was used where each image is divided into 16 regions with radius $\mathrm{R}=1$. $\mathrm{kNN}, \mathrm{SVM}$ and $\mathrm{NN}$ classifiers were used for the classification stage.

\subsection{The Databases Used}

For evaluating of the proposed approach extensive experiments were carried out on 3 different databases. The first database, The JAFFE database [31] contains 213 images of 10 different females each with 7 basic facial expressions namely; anger (AN), disgust (DI), fear (FE), happy (HA), sad (SA), surprise (SU) and neutral (NE). Resolution of the images is $256 \times 256$ pixels. Examples of images from JAFFE database are shown in Fig. 6. 


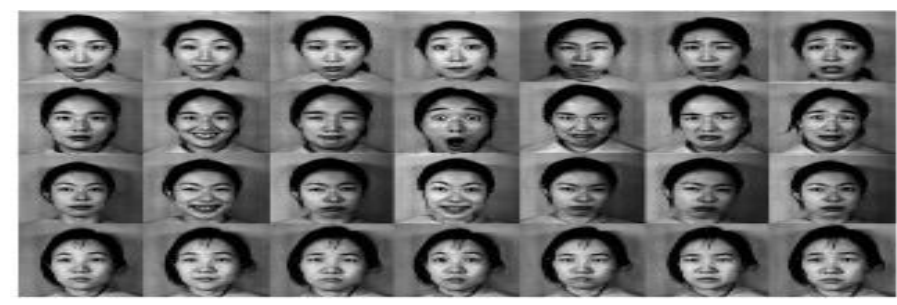

Fig. 6. Examples of images from the JAFFE database.

The second database, Cohn-Kanade (CK) database [32] contains 486 image sequences posed across 97 subjects. Each of the sequence contains images from onset (neutral frame) to peak expression (last frame) and a resolution of $640 \times 490$ pixels. The images consist of 12-16 frames. The ages of the subjects ranged from 18-30 years of which $65 \%$ were female and $35 \%$ were male. Images were resized to $256 \times 256$ pixels. Fig. 7 shows examples of image sequences in Cohn-Kanade database.

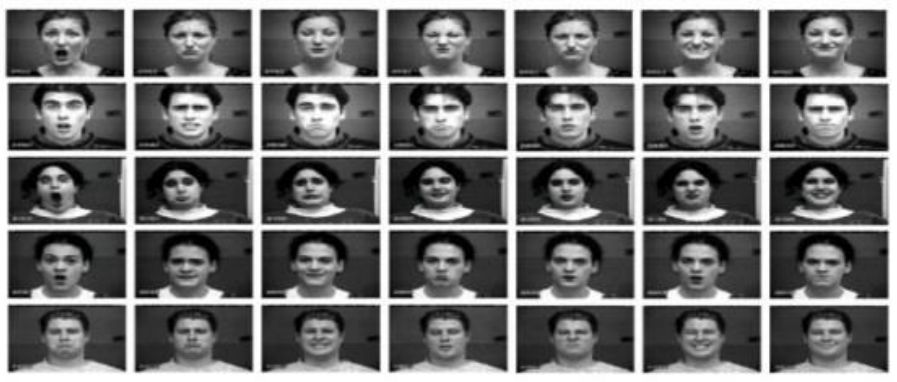

Fig. 7. Examples of Images from CK Database.

Third database is Mevlana University Facial Expression (MUFE) database [33]. The facial expressions of 15 students were taken at Mevlana University. The database contains 630 facial images of 15 individuals with $80 \%$ males and $20 \%$ females. Each of the basic 7 facial expressions was recorded 6 times for each subject ( 2 frontal images, 2 images with subject looking slightly to the right of the camera and 2 images with subject looking slightly to the left of the camera). All the images in the database were manually cropped and resized to $256 \times 256$ pixels. Fig. 8 shows examples of images from MUFE database.

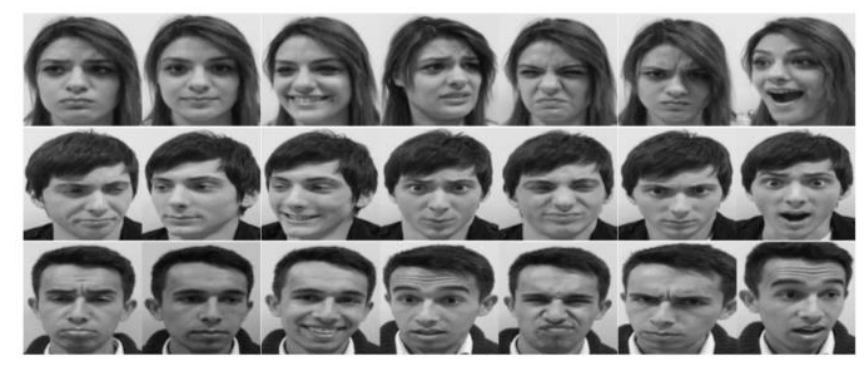

Fig. 8. Examples of images from MUFE database.

\section{Simulation Results \& Discussions}

The proposed approach was implemented on JAFFE, MUFE and CK facial expression databases using person-dependent approach and 10-fold cross validation under MATLAB environment. For JAFFE database, 137 of the images were used as training set while 76 images used as testing set. On the other hand, for MUFE database, 50\% of the images were used for both training and testing set. 97 
subjects from CK database were used making a total of 2716 images with four images per expression per subject. Here three images per subject per expression were used for training, thus, 2037 training images, while one image per subject per expression was used for testing, which gives a total of 679 testing images. GWT and DT-CWT were implemented in three different scenarios. First scenario was implemented using the generated feature vectors directly, while in second and third scenarios feature vectors lengths were reduced using PCA or LBP algorithms before classification. Neural networks with multilayer feed-forward architecture and back propagation learning algorithm and SVM with radial basis function (RFB) kernel were used in our implementation. The results of all the experiments are given in the following tables. Table 1 shows the recognition performance using the 3 facial expression databases. Comparisons between kNN and SVM classifiers are shown for GWT, GWT+PCA and GWT+LBP approaches. Comparisons were conducted on each facial expression separately. The average recognition performance for each database was also recorded in the last row of the table. GWT+PCA+SVM approach recorded the best average performance among other approaches. This result was consistent for the three databases used.

Table 1. Facial expression recognition for GWT using three facial expression databases.

\begin{tabular}{|c|c|c|c|c|c|c|}
\hline \multirow{3}{*}{ 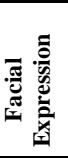 } & \multicolumn{6}{|c|}{ GWT / GWT+PCA / GWT+LBP } \\
\hline & \multicolumn{2}{|c|}{ JAFFE } & \multicolumn{2}{|c|}{ MUFE } & \multicolumn{2}{|c|}{ CK } \\
\hline & kNN & SVM & kNN & SVM & kNN & SVM \\
\hline$\overline{\mathbf{A N}}$ & $91.03 / 82.10 / 90.95$ & $89.88 / 99.56 / 90.11$ & $80.93 / 92.21 / 68.76$ & $93.20 / 92.42 / 69.04$ & $76.98 / 92.20 / 96.84$ & $96.86 / 96.97 / 92.07$ \\
\hline DI & $82.89 / 67.21 / 92.11$ & $91.23 / 91.04 / 91.00$ & $90.79 / 88.65 / 80.23$ & $89.12 / 92.28 / 85.15$ & 96.86 / 96.97 / 96.42 & $87.01 / 96.97$ / 96.53 \\
\hline $\mathbf{F E}$ & $75.13 / 58.24 / 75.09$ & $91.88 / 91.87$ / 92.30 & $80.32 / 80.02 / 66.79$ & $77.78 / 79.00 / 79.92$ & $87.30 / 91.64$ / 76.98 & $82.64 / 91.53 / 87.12$ \\
\hline HA & $90.05 / 69.91 / 100.0$ & $83.13 / 92.17 / 91.84$ & $78.76 / 90.28 / 78.18$ & 91.34 / 92.33 / 78.31 & $86.88 / 87.17 / 87.00$ & $92.31 / 96.97$ / 96.97 \\
\hline $\mathbf{S A}$ & $91.19 / 63.30 / 81.93$ & $81.92 / 100.0 / 99.95$ & $83.23 / 91.40 / 83.88$ & $90.65 / 91.17 / 82.89$ & 96.53 / $96.97 / 88.30$ & 89.77 / 87.20 / 89.40 \\
\hline $\mathbf{S U}$ & $90.08 / 89.90 / 90.22$ & $100.0 / 100.0 / 90.89$ & $88.69 / 86.15 / 71.20$ & $89.56 / 97.64$ / 75.19 & $87.00 / 96.97 / 86.64$ & 96.53 / 96.97 / 96.97 \\
\hline NE & $100.0 / 99.94 / 100.0$ & $100.0 / 99.76 / 100.0$ & 82.37 / 93.37 / 76.09 & $92.76 / 96.78 / 82.11$ & 96.97 / 96.84 / 96.97 & 96.97 / 96.97 / 96.75 \\
\hline Avg & $88.62 / 75.80 / 90.04$ & $91.15 / 96.33 / 93.73$ & 83.58 / 88.87 / 75.02 & $89.20 / 91.66 / 78.95$ & $89.79 / 94.12$ / 89.88 & $91.73 / 94.80 / 93.69$ \\
\hline
\end{tabular}

Same experiment setup was carried out in Table 2 with the replacement of GWT with DT-CWT. Comparisons between kNN and NN classifiers are shown for DT-CWT, DT-CWT+PCA and DT$\mathrm{CWT}+\mathrm{LBP}$ approaches. The average recognition performance for each database was also recorded in the last row of the table. GWT+PCA+NN approach recorded the best average performance among other approaches. In general, kNN classifier, as expected, recorded lower performances than SVM and NN classifiers for the three databases in all scenarios.

Table 2. Facial expression recognition for DT-CWT using three facial expression databases.

\begin{tabular}{|c|c|c|c|c|c|c|}
\hline \multirow{3}{*}{ 苞 } & \multicolumn{6}{|c|}{ DT-CWT / DT-CWT+PCA / DT-CWT+LBP } \\
\hline & \multicolumn{2}{|c|}{ JAFFE } & \multicolumn{2}{|c|}{ MUFE } & \multicolumn{2}{|c|}{ CK } \\
\hline & kNN & $\mathbf{N N}$ & kNN & $\mathbf{N N}$ & kNN & $\mathbf{N N}$ \\
\hline$\overline{\mathbf{A N}}$ & $99.78 / 100.0 / 80.45$ & $57.56 / 91.44 / 100.0$ & $80.16 / 67.76 / 64.35$ & 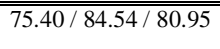 & $85.60 / 94.46 / 97.05$ & $8787.50 / 87.64 / 92.05$ \\
\hline DI & $91.23 / 73.34 / 90.56$ & $99.67 / 90.56 / 92.37$ & $82.26 / 82.23 / 80.12$ & $59.41 / 89.95 / 89.32$ & $96.59 / 91.05 / 94.68$ & $96.51 / 97.05 / 96.44$ \\
\hline $\mathbf{F E}$ & $83.12 / 74.78 / 67.67$ & $76.34 / 92.13 / 80.17$ & $76.23 / 52.78 / 70.67$ & $76.66 / 79.51 / 82.83$ & 94.34 / $94.49 / 94.17$ & $87.76 / 87.61 / 82.93$ \\
\hline HA & $74.78 / 57.89 / 66.56$ & $52.88 / 99.56 / 94.78$ & $79.36 / 62.13 / 71.16$ & $50.25 / 99.94 / 77.05$ & $99.75 / 95.06 / 99.95$ & $97.35 / 97.04 / 96.95$ \\
\hline SA & $64.33 / 73.34 / 45.02$ & $65.17 / 100.0 / 99.67$ & $80.21 / 76.18 / 80.25$ & $59.84 / 78.37 / 88.47$ & $95.16 / 91.63 / 92.53$ & 96.94 / 96.47 / 96.57 \\
\hline $\mathbf{S U}$ & $99.67 / 100.0 / 80.45$ & 77.45 / 99.89 / 90.56 & $84.03 / 76.23 / 79.68$ & $71.85 / 87.05 / 77.29$ & 96.15 / 99.95 / 99.71 & 86.89 / 96.69 / 97.29 \\
\hline $\mathbf{N E}$ & $99.78 / 90.15 / 100.0$ & $59.78 / 91.39 / 85.15$ & $86.56 / 63.56 / 76.33$ & $48.96 / 73.90 / 73.75$ & $95.04 / 84.10 / 91.25$ & $96.78 / 97.00 / 96.84$ \\
\hline Avg & $87.53 / 81.36 / 75.82$ & 69.84 / $95.00 / 91.81$ & $81.26 / 68.70 / 74.65$ & $63.19 / 84.75$ / 81.38 & $91.62 / 89.92 / 92.58$ & $92.82 / 94.21 / 94.15$ \\
\hline
\end{tabular}


In Fig. 9, comparisons with the well-known feature extraction algorithms scale-invariant feature transform (SIFT) [34] and speeded up robust features (SURF) [35] are performed on the three databases. In general, the average numbers of key points obtained for an image are 36 and 16 key points using SIFT and SURF, respectively. SIFT performance was slightly better than SURF for the three databases. Still, GWT as a feature extractor recorded the best performance among other algorithms for all databases.

Comparisons with other works conducted on the JAFFE database is shown in Table 3. To the best of our knowledge, all the algorithms available in this table, except algorithm in [39], used persondependent approach in their simulations. The number inside the parentheses next to recognition rates indicates the number of facial expressions used. If the number is equal to 6 it means the neutral facial expression was neglected.

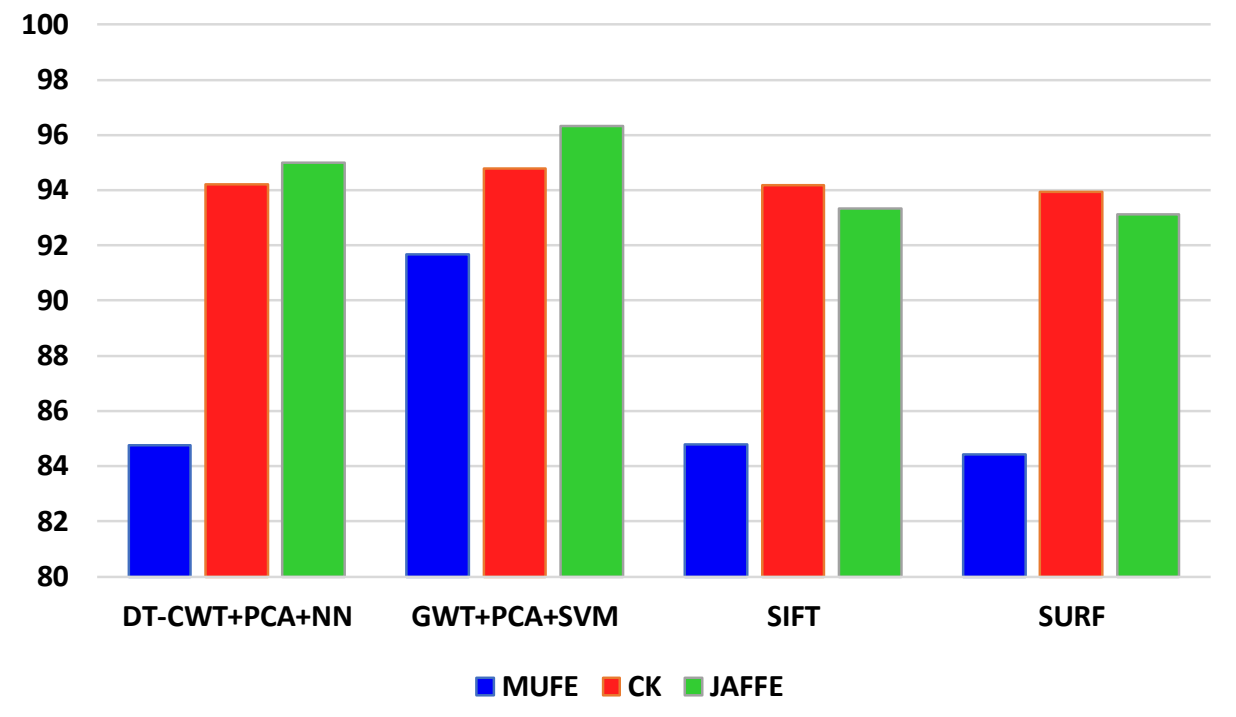

Fig. 9. Facial expression recognition comparison for MUFE, CK and JAFFE databases using different algorithms.

Fig. 10 shows the general average recognition performances of the 7 facial expressions form table 1 and table 2. Averages were calculated using all the algorithms and databases results. It is clearly obvious that disgust (DI), surprise (SU) and neutral (NE) expressions got the highest average recognition performances $(>89 \%)$. Fear $(\mathrm{FE})$ expression recorded the worst average recognition performance $(\sim 81 \%)$. Justification of low recognition performance of fear expression might be that sometimes fear expression can be close to surprise expression. 


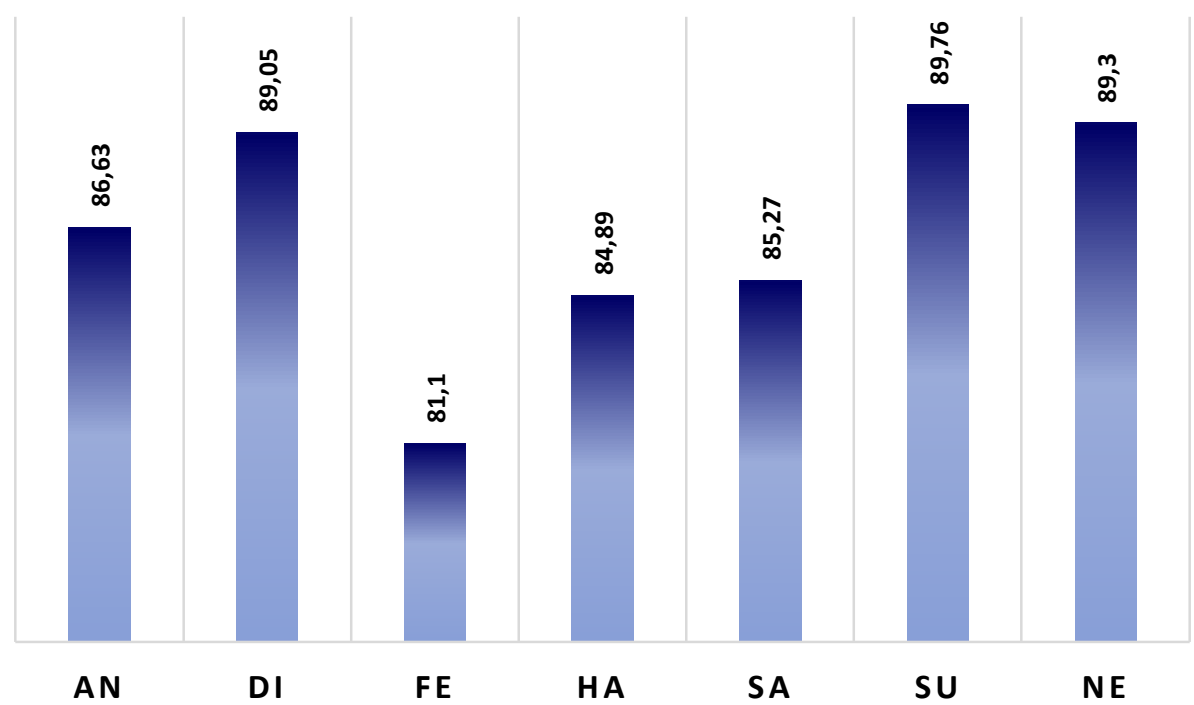

Fig. 10. Average recognition performances of the 7 facial expressions from all applied algorithms and databases.

Table 3. Facial expression recognition performance comparisons for JAFFE database (the listed results are the best recorded results in the respective articles)

\begin{tabular}{lc}
\hline Algorithm & Recognition rates \\
\hline Lyons et al. [34] & $92.00 \%(7)$ \\
Zhang et al. [35] & $90.10 \%(7)$ \\
Buciu et al. [36] & $90.34 \%(7)$ \\
Dubuisson et al. [37] & $87.60 \%(6)$ \\
Shinohara \& Otsu [38] & $69.40 \%(7)$ \\
Kung et al. [39] & $68.85 \%(6)$ \\
Happy \& Routray [40] & $87.43 \%(6)$ \\
Shih et al. [41] & $95.71 \%(7)$ \\
DT-CWT+PCA+NN & $95.00 \%(7)$ \\
GWT+PCA+SVM & $96.33 \%(7)$ \\
\hline
\end{tabular}

\section{Conclusions}

This work investigated facial expression recognition problem using GWT and DT-CWT with kNN, SVM and NN classifiers using person-dependent approach. PCA and LBP algorithms were used to reduce the dimensions of the feature vectors generated using GWT and DT-CWT. GWT+PCA with SVM classifier recorded the best average recognition rates of $96.33 \%, 91.66 \%$ and $94.8 \%$ for JAFFE, MUFE and CK databases, respectively. Experimental results obtained demonstrated the high performance and advantage of using DT-CWT in FER problem. The recorded best average recognition rates DT-CWT with NN are 95\%, 84.75\% and 94.21\% for JAFFE, MUFE and CK 
databases, respectively. The overall performance of NN and SVM were, as expected, better than the $\mathrm{kNN}$ in all scenarios. Also, the importance of applying dimensionality reduction on the feature vectors before classification stage was realized, where in most experiments the performance increased after applying dimensionality reduction. Finally, it could be seen that the accuracy of the proposed MUFE database is lower than the other databases; firstly, this is due to the fact that MUFE database consist of not only frontal face images but also from slightly left and right orientations of the faces. This is unlike JAFFE and CK where only frontal face images are recorded. Secondly, the participants were not professional actors, hence didn't always give as perfect expressions as desired.

\section{References}

[1] Niu Z., Qiu X., Facial expression recognition based on weighted principal component analysis and support vector machines, IEEE 3rd International Conference on Advanced Computer Theory and Engineering, 174-178, 2010.

[2] Song K.T., Chen Y.W., A design for integrated face and facial expression recognition, 37th Annual conference on IEEE Industrial Electronics Society, 4306-4311, 2011.

[3] Vinciarelli A., Pantic M., Bourlard H., Social signal processing survey of an emerging domain, Image and Vision Computing, 1743-1759, 2009.

[4] Lin D., Facial expression classification using PCA and hierarchical radial basis function network, Journal of Information Science and Engineering, 22(5), 1033-1046, 2006.

[5] Mehrabian A., Communication without words, Psychology Today, 2(4), 53-56, 1968.

[6] Ekman P., Friesen W., Facial action coding system: a technique for the measurement of facial movements, Consulting Psychologists Press, California, 1978.

[7] Yacoob Y., Davis L.S., Recognizing human facial expression from long image sequences using optical flow, IEEE Transactions on Pattern Analysis and Machine Intelligence, 18(6), 636-642, 1996.

[8] Brunelli R., Poggio T., Face recognition: features vs. templates, IEEE Transaction on Pattern Analysis and Machine Intelligence, 15(10), 1042-1053, 1993.

[9] Eleyan A., Demirel H., Performance comparison among complex wavelet transforms based face recognition systems, Image Processing and Communication Conference, AISC84, 201209, 2010.

[10] Turk M., Pentland A., Eigenfaces for recognition, Journal of Cognitive Neuroscience, 3(1), 7186, 1991.

[11] Abdulrahman M., Gwadabe T. R., Abdu F. J., Eleyan A., Gabor wavelet transform based facial expression recognition using PCA and LBP. In IEEE 22nd Signal Processing and Communications Applications Conference (SIU), 2265-2268, 2014.

[12] Lee T. S., Image representation using 2d Gabor wavelets, IEEE Transactions on Pattern Analysis and Machine Intelligence, 18(10), 959-971, 1996.

[13] Lyons M. J., Budynek J., Akamatsu S., Automatic classification of single facial images, IEEE Transactions on Pattern Analysis and Machine Intelligence, 21(12), 1357-1362, 1999.

[14] Gu W., Xiang C., Venkatesh Y., Huang D., Lin H., Facial expression recognition using radial encoding of local Gabor features and classifier synthesis, Pattern Recognition, 45(1), 80-91, 2012.

[15] Kong W. K., Zhang D., W. Li, Palm print feature extraction using 2-d Gabor filters, Pattern Recognition Society, 2339-2347, 2003.

[16] Karimimehr N., Sharazi A. A. B., Kashavar B. M., Finger print image enhancement using Gabor wavelet transform, 18th Iranian Conference on Electrical Engineering Proceeding, 316$320,2010$. 
[17] Kingsbury N. G., The dual-tree complex wavelet transform: a new efficient tool for image restoration and enhancement, European Signal Processing Conference, 319-322, 1998.

[18] Eleyan A., Demirel H., Ozkaramanli H., Face recognition using dual-tree wavelet transform, IEEE International Symposium on Signal Processing and Information Technology, 7-11, 2008.

[19] Liu C.C., Dai D.Q., Face recognition using dual-tree complex wavelet features, IEEE Transactions on Image Processing, 18(11), 2593-2599, 2009.

[20] Sun Y.H., Du M.H., Face detection using dt-cwt on spectral histogram. IEEE Proceedings, Fifth International Conference on Machine Learning and Cybernetics, 3637-3642, 2006.

[21] Wang Y., Dual-tree complex wavelet transform based local binary pattern weighted histogram method for palm print recognition, Computing and Informatics, 28, 299-318, 2009.

[22] Zhang S., Tang T., Wu C., Xi N., Wang G., A novel image denoising method using independent component analysis and dual-tree complex wavelet transform, 6th International Conference on Wireless Communications Networking and Mobile Computing, 1-4, 2010.

[23] Chen G. Y., Xie W. F., Pattern recognition using dual-tree complex wavelet features and svm, Canadian Conference on Electrical and Computer Engineering, 2053-2056, 2005.

[24] Eleyan A., Özkaramanli H., Demirel H., Complex wavelet transform-based face recognition, EURASIP Journal on Advances in Signal Processing, 2008(1), 1-13, 2009.

[25] Li Y., Ruan Q., An G., Li X., Facial expression recognition based on the dual-tree complex wavelet transform and supervised spectral analysis, IEEE 10th International Conference on Signal Processing (ICSP), 1301-1304, 2010.

[26] Daugman J., Uncertainty relation for resolution in space, spatial frequency and orientation optimized by two-dimensional visual cortical filters. Journal of the Optical Society of America A, 2(7), 1160-1169, 1985.

[27] Struc V., Pavesic N., Gabor-based kernel partial least squares discrimination features for face recognition, Informatica, 20(1), 115-138, 2009.

[28] Liu C., Wechsler H., Gabor feature based classification using the enhanced fisher linear discriminant model for face recognition, IEEE Transactions on Image Processing, 11(4), 467476, 2002.

[29] Shen L., Bai L., Fairhurst M., Gabor wavelets and general discriminant analysis for face identification and verification, Image and Vision Computing, 25(5), 553-563, 2007.

[30] Ojala T., Pietikainen M., Maenpaa T., Multi-resolution gray scale and rotation invariant texture analysis with local binary patterns, IEEE Transactions on Pattern Analysis and Machine Intelligence, 24(7), 971-987, 2010.

[31] Lyons M. J., Akemastu S., Kamachi M., Gyoba J., Coding facial expressions with Gabor wavelets, 3rd IEEE International Conference on Automatic Face and Gesture Recognition, 200-205, 1998.

[32] Lucey P., Cohn J.F., Kanade T., Saragih J., Ambadar Z., Matthews I., The extended CohnKande dataset $(\mathrm{CK}+)$ : A complete facial expression dataset for action unit and emotionspecified expression, 3rd IEEE Workshop on CVPR for Human Communicative Behaviour Analysis, 94-101, 2010.

[33] Abdulrahman M., Eleyan A., Facial expression recognition using support vector machines, 23rd IEEE International Signal Processing and Communication Applications Conference (SIU), 276-279, 2015.

[34] Lyons M., Budynek J., Akamatsu S., Automatic classification of single facial images, IEEE Transaction on Pattern Analysis and Machine Intelligence, 21(12), 1357-1362. 1999

[35] Zhang Z., Lyons M., Schuster M., Akamatsu S., Comparison between geometry based and Gabor-wavelets-based facial expression recognition using multi-layer perceptron, IEEE International Conference on Automatic Face and Gesture Recognition, 454-459. 1998

[36] Buciu I., Kotropoulos C., Pitas I., ICA and Gabor representation for facial expression recognition, IEEE International Conference on Image Processing (ICIP), 855-858. 2003. 
[37] Dubuisson S., Davoine F., Masson M., A solution for facial expression representation and recognition, Signal Processing: Image Communication, 17, 657-673. 2002

[38] Shinohara Y., Otsu N., Facial expression recognition using fisher weight maps, IEEE International Conference on Automatic Face and Gesture Recognition, 499-504. 2004.

[39] Kung H. W., Tu Y. H., Hsu C. T., Dual subspace nonnegative graph embedding for identityindependent expression recognition, IEEE Transactions on Information Forensics and Security, 10(3), 626-639, 2015.

[40] Happy S. L., Routray A., Robust facial expression classification using shape and appearance features, 8th International Conference on Advances in Pattern Recognition (ICAPR), 1-5. 2015.

[41] Shih F. Y., Chuang C., Wang P. S., Performance comparisons of facial expression recognition in JAFFE database, International Journal of Pattern Recognition and Artificial Intelligence, 22(3), 445-459, 2008. 